Bundesgesundheitsbl 2021 · 64:1483-1491 https://doi.org/10.1007/s00103-021-03448-0 Eingegangen: 16. Juni 2021

Angenommen: 6. Oktober 2021

Online publiziert: 3. November 2021

c c Der/die Autor(en) 2021

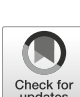

Roma Thamm • Nina Buttmann-Schweiger • Julia Fiebig • Christina Poethko-Müller · Franziska Prütz - Giselle Sarganas . Hannelore Neuhauser

Abteilung Epidemiologie und Gesundheitsmonitoring, Robert Koch-Institut, Berlin, Deutschland

\title{
SARS-CoV-2-Seroprävalenz bei Kindern und Jugendlichen in Deutschland - ein Überblick
}

lide Daten zur Gesamtheit der Infizierten die zentrale Grundlage zur Einschätzung der Lage und darauf aufbauend der Umsetzung geeigneter Schutzmaßnahmen. Serologische Untersuchungen auf SARSCoV-2-Antikörper ergänzen die Meldestatistik laborbestätigter Fälle um Informationen zum Anteil der unerkannten Fälle. Dadurch ergibt sich die Untererfassung oder Dunkelziffer. Unter Hinzunahme serologischer Ergebnisse kann zudem der Anteil Verstorbener unter den jemals Erkrankten (Infection Fatality Rate, IFR) genauer bestimmt werden, unter Einschluss auch nichtgemeldeter und asymptomatischer Infektionen.

Asymptomatische oder nur mild symptomatische SARS-CoV-2-Infektionen kommen bei Kindern und Jugendlichen deutlich häufiger vor als bei Erwachsenen [4-8]. Vor diesem Hintergrund sind seroepidemiologische Untersuchungen gerade bei Kindern und Jugendlichen hoch relevant. Wichtig sind solche Studien auch angesichts aktueller Untersuchungen, die darauf hinweisen, dass selbst asymptomatische SARS-CoV-2Infektionen $\mathrm{zu}$ ernst $\mathrm{zu}$ nehmenden gesundheitlichen Folgen wie dem pädiatrischen Multiorgan-Immunsyndrom (PMIS) führen können [9, 10]. Der vorliegende Beitrag fasst bisherige Ergebnisse zur SARS-CoV-2-Prävalenz aus seroepidemiologischen Studien in Deutschland zusammen, die sich auf Kinder und Jugendliche konzentrieren. Er ergänzt die bereits vorliegende Übersicht von Ergebnissen zur Seroprävalenz in Zufallsstichproben der Allgemeinbevölkerung mit Fokus auf Erwachsene und bei Blutspendenden in Deutschland [11].

\section{Material und Methoden}

Seit dem Frühjahr 2020 wird im Robert Koch-Institut eine fortlaufende systematische Recherche von seroepidemiologischen Studien in Deutschland sowie deren Ergebnissen durchgeführt und der Kontakt zu den jeweiligen Studienleitungen hergestellt. Die Recherche umfasst Studienregister, Literaturdatenbanken, Preprint-Veröffentlichungen und Medienberichte. Eine ausführliche Beschreibung dieser Recherche inklusive der Suchstrategie und Erläuterungen zu methodischen Unterscheidungsmerkmalen seroepidemiologischer Studien in Bezug auf Stichprobenrahmen und Gütekriterien der eingesetzten Antikörpertests ist im Journal of Health Monitoring publiziert [12]. Aus der Recherchetätigkeit resultierte eine seit dem 01.07.2020 verfügbare Webseite in deutscher ${ }^{1}$ und englischer ${ }^{2}$ Sprache. Die regelmäßig aktualisierte Webseite führt Angaben zum Studiendesign sowie Links zu veröffentlichten Studienprotokollen, Studienwebseiten und Ergebnismitteilungen bzw. Publikationen auf. Die Übersicht gruppiert mit Stand vom 01.09.2021 in Antikörperstudien in der Allgemeinbevölkerung (inklusive Studien in bestehenden Kohorten und bei Blutspendenden) und in Studien in

\footnotetext{
1 www.rki.de/covid-19-ak-studien.

2 www.rki.de/covid-19-serostudies-germany.
} 


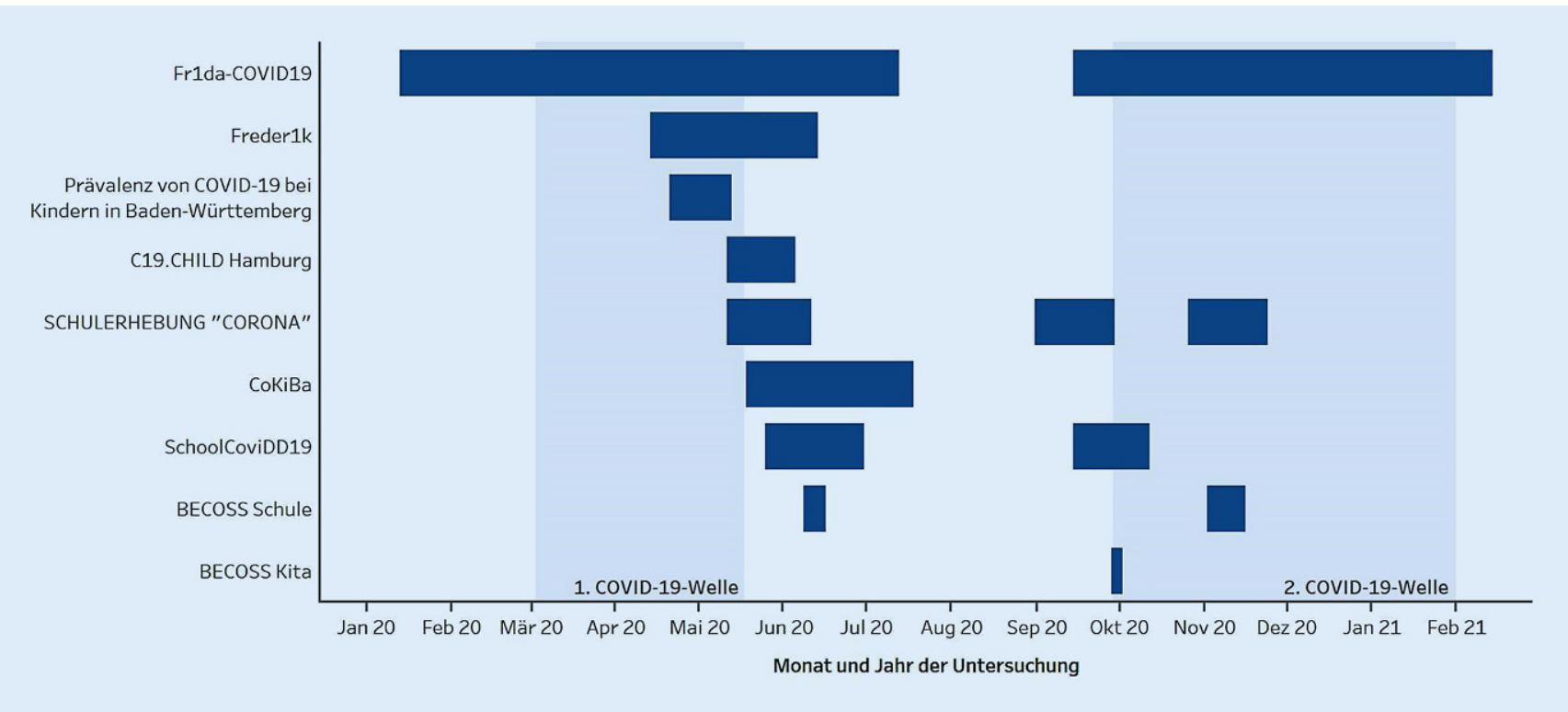

Abb. 1 ॥ Untersuchungszeiträume seroepidemiologischer SARS-CoV-2-Studien mit Fokus auf Kinder und Jugendliche in Deutschland. (Eigene Abbildung)

besonderen Bevölkerungsgruppen, z. B. bei Beschäftigten in Krankenhäusern, in Betreuungseinrichtungen oder anderen Betrieben. $\mathrm{Zu}$ den Studien, die sowohl Erkenntnisse über Antikörper in der Allgemeinbevölkerung als auch in besonderen Bevölkerungsgruppen gewinnen wollen, zählen vor allem Untersuchungen in Kitas, Kindergärten oder Schulen, bei denen sowohl die Kinder oder Jugendlichen als auch die Beschäftigten der Betreuungseinrichtung auf Antikörper getestet werden. Eine interaktive Deutschlandkarte zeigt, wo in Deutschland seroepidemiologische Studien durchgeführt werden.

Für belastbare Aussagen zur Seroprävalenz bei Kindern und Jugendlichen in verschiedenen Altersgruppen reicht die Einbeziehung von Kindern und Jugendlichen in Studien mit Stichproben der Allgemeinbevölkerung oft nicht aus. Daher wurden bisher zumeist Studien bei Kitakindern sowie Schülerinnen und Schülern durchgeführt, aber auch bei Kindern und Jugendlichen, die Vorsorgeuntersuchungen in Kinderarztpraxen in Anspruch nehmen oder die in Kinderkliniken behandelt wurden. Im vorliegenden Beitrag werden Metadaten der einzelnen Studien, wie beispielsweise Studienort oder -region und untersuchte Stichprobe, sowie ihre Ergebnisse tabellarisch dargestellt.

\section{Ergebnisse}

Mit Stand 17.09.2021 haben wir 16 deutsche seroepidemiologische Studien, die sich auf Kinder und Jugendliche konzentrieren, gefunden. Für 9 dieser Studien liegen Ergebnisse vor, die als Studienbericht (1), Pressemitteilung (1), PreprintPublikation (1) oder als bereits peer-reviewte Publikation (6) veröffentlicht wurden. In • Abb. 1 sind die einzelnen Untersuchungszeiträume dieser 9 seroepidemiologischen Studien dargestellt. Um die Studien leichter im Verlauf des Pandemiegeschehens in Deutschland verorten zu können, wurden die Zeiträume der ersten und zweiten COVID-19-Welle zusätzlich visualisiert [13].

Bereits im Januar 2020 startete mit der Fr1da-COVID19-Studie die erste Studie zur Ermittlung der SARS-CoV-2-Seroprävalenz bei Kindern in Deutschland [14]. Diese konnte vor allem deshalb so schnell realisiert werden, weil die Stichprobe auf einer bereits bestehenden Kohortenstudieninfrastruktur basiert, und zwar der Frlda-Studie in Bayern zur Früherkennung des Typ-1-Diabetesrisikos bei Kindern, die Vorsorgeuntersuchungen wahrnehmen. Ab April 2020 wurden Antikörpertests auch bei einer Unterstichprobe der Freder1k-Studie in Bayern, dem Pendant zur Fr1da-Studie bei Neugeborenen, eingesetzt [15]. Über den Zeitraum Mai bis September 2020 folgten weitere 7 Studien, zum Teil mit Nachuntersuchungen, in verschiedenen Regionen Deutschlands [16-23]. Diese Studien wurden zumeist auf der Basis von Freiwilligenstichproben konzipiert, auch bekannt als Nichtzufallsstichproben oder Convenience Samples.

In den - Tab. 1 und 2 sind Metadaten der seroepidemiologischen Studien mit Stichproben gelistet, die auf der Teilnahme an Vorsorgeuntersuchungen, beziehungsweise auf Freiwilligenstichproben in verschiedenen Settings basieren. Die Metadaten beinhalten Angaben zum Studienort oder zur Studienregion, zum Stichprobenrahmen, zum Untersuchungszeitraum, zur untersuchten Stichprobe (Anzahl Teilnehmende und Altersbereich) und zu den eingesetzten Antikörpertests. Für jede Studie sind die Ergebnisse zur berichteten SARSCoV-2-Seroprävalenz mit 95\%-Konfidenzintervall (KI) angegeben. Für die meisten Studien sind neben der GesamtSARS-CoV-2-Seroprävalenz auch stratifizierte Seroprävalenzen für mehrere Altersgruppen angegeben.

Die Ergebnisse der Studien lassen sich nur schwer übergreifend zusammenfassen. Gründe dafür sind verschiedene Altersbereiche, d.h. Kitaalter (bis ca. 5 Jahre), Grundschulalter (6 bis ca. 10 Jahre) und Jugendalter (11 bis 
Bundesgesundheitsbl $2021 \cdot 64: 1483-1491$ https://doi.org/10.1007/s00103-021-03448-0

(c) Der/die Autor(en) 2021

R. Thamm · N. Buttmann-Schweiger · J. Fiebig · C. Poethko-Müller · F. Prütz · G. Sarganas · H. Neuhauser

\section{SARS-CoV-2-Seroprävalenz bei Kindern und Jugendlichen in Deutschland - ein Überblick}

\section{Zusammenfassung}

Hintergrund. SARS-CoV-2-Antikörperstudien ergänzen und erweitern die Erkenntnisse aus der Meldestatistik laborbestätigter COVID-

19-Fälle um Informationen zu unentdeckten Fällen.

Ziel der Arbeit. Der vorliegende Beitrag fasst bisherige Ergebnisse zur SARS-CoV-2Prävalenz aus seroepidemiologischen Studien in Deutschland zusammen, die sich auf Kinder und Jugendliche konzentrieren, und ergänzt die bereits vorliegende Übersicht zur Seroprävalenz bei Erwachsenen und speziell bei Blutspendenden in Deutschland. Material und Methoden. Die Ergebnisse der Übersichtsarbeit beruhen auf einer fortlaufenden systematischen Recherche in
Studienregistern, Literaturdatenbanken, von Preprint-Veröffentlichungen und Medienberichten seroepidemiologischer Studien in Deutschland sowie deren Ergebnissen. Ergebnisse. Mit Stand 17.09.2021 sind uns 16 deutsche seroepidemiologische Studien, die sich auf Kinder und Jugendliche konzentrieren, bekannt geworden. Für 9 dieser Studien liegen Ergebnisse vor. Für fast alle untersuchten Settings lag die SARSCoV-2-Seroprävalenz für Kinder im Kita- und Grundschulalter in der ersten COVID-19-Welle deutlich unter $1 \%$ und für Jugendliche unter $2 \%$. Im Verlauf der Pandemie wurden höhere Seroprävalenzen von bis zu $8 \%$ für Kinder im Grundschulalter ermittelt.
Diskussion. Ergebnisse von SARS-CoV2-Antikörperstudien bei Kindern und Jugendlichen in Deutschland liegen bislang erst in geringem Umfang und basierend auf lokal-regionalen, nichtrepräsentativen Stichproben vor. In künftigen Studien gilt es, einerseits abzuschätzen, welcher Anteil der Kinder und Jugendlichen bereits eine Infektion hatte oder geimpft ist. Zum anderen gilt es, die Verbreitung körperlicher und psychischer Beeinträchtigungen im Nachgang einer Infektion zu untersuchen.

Schlüsselwörter

Coronavirus · Antikörper · Kinder und Jugendliche · Immunstatus · Serologie

\section{Seroprevalence of SARS-CoV-2 among children and adolescents in Germany-an overview}

\section{Abstract}

Background. SARS-CoV-2 serologic studies complement and expand findings from confirmed COVID-19 cases through identification of undetected cases.

Objectives. This article summarizes previous results on SARS-CoV-2 prevalence from seroepidemiological studies in Germany focusing on children and adolescents and complements the already existing overview on seroprevalence in adults from general population samples and especially blood donors in Germany.

Methods. The results are based on an ongoing systematic search in study registries, in literature databases, of preprint publications, and of media reports of seroepidemiological studies in Germany and their results.

Results. As of 17 September 2021, we are aware of 16 German seroepidemiological studies focusing on children and adolescents. Results are available for nine of these studies. For almost all settings studied, SARS-CoV-2 seroprevalence was well below $1 \%$ for preschool and elementary school children in the first COVID-19 wave and below $2 \%$ for adolescents. As the pandemic progressed, higher seroprevalences of up to $8 \%$ were found in elementary school children. Discussion. Results of SARS-CoV-2 antibody studies in children and adolescents in
Germany are scarce so far and are based on non-representative samples at local or regional level. In future studies, it is necessary on the one hand to estimate which proportion of children and adolescents has already either had an infection or has been vaccinated. On the other hand, it is important to investigate physical and mental health impairments that occur after an infection.

\section{Keywords}

Coronavirus · Antibodies · Children and adolescents · Immunity · Serology
18 Jahre), eine Rekrutierung in Orten bzw. Regionen mit sehr unterschiedlich hohem Infektionsgeschehen, der meist nicht zufällige Stichprobenzugang mit verschiedenen Selektionseffekten sowie die verschiedenen COVID-19-Wellen (erste Jahreshälfte 2020 und zweite Jahreshälfte 2020 bis Februar 2021). Dennoch lag trotz aller Verschiedenheiten für fast alle untersuchten Settings die SARS-CoV-2-Seroprävalenz für Kinder im Kita- und Grundschulalter in der ersten COVID-19-Welle deutlich unter $1 \%$ und für Jugendliche unter $2 \%$. Alle seroepidemiologischen Studien, die Nachuntersuchungen beziehungsweise
2. Untersuchungswellen in der zweiten Jahreshälfte 2020 bis Februar 2021 durchgeführt haben, ermittelten höhere SARS-CoV-2-Seroprävalenzen als in der ersten Pandemiewelle. Altersstratifiziert liegen aus der Fr1da-Studie aus Bayern Ergebnisse für Kinder im Kita- und Grundschulalter vor. Für die Gesamtaltersgruppe der 1- bis 10-Jährigen ist die Seroprävalenz von $0,7 \%$ auf $3,9 \%$ gestiegen. Am Ende dieser zweiten Beprobungsrunde im JanuarFebruar 2021 betrug die Seroprävalenz für Kinder im Kitaalter 5,6\% und für Kinder im Grundschulalter 8,4\%. Für Kinder und Jugendliche berichtet die
Berliner BECOSS-Studie einen Anstieg der SARS-CoV-2-Seroprävalenz von Juni 2020 bis November 2020 von $1,3 \%$ auf 2,0\%.

Erst in einer Studie, der Fr1da-Studie, wurde aus der ermittelten Seroprävalenz auf die Untererfassung von SARS-CoV2-Infektionen bei Kindern geschlossen. Laut Fr1da lag die Untererfassung in der ersten Jahreshälfte 2020 bei Faktor 6 und damit leicht höher als in den im ersten Halbjahr 2020 durchgeführten Studien bei Erwachsenen, in denen eine Untererfassung um den Faktor 4 bis 5 gefunden wurde. Das heißt, die Ergebnisse der Studie legen nahe, dass es etwa 6-mal so 
Tab. 1 Seroepidemiologische Studien bzgI. SARS-CoV-2 bei Kindern und Jugendlichen mit Stichproben basierend auf Teilnahme an Vorsorgeuntersuchungen

\section{Antikörpertest}

Alter in

Seroprävalenz (95\%-

\begin{tabular}{ll} 
Studie & Stichprobenrahmen \\
& \\
\hline Fr1da-COVID19: Mes- & Einladung aller Kinder, die \\
sung der Entwicklung & am Diabetes-Typ-1-Scree- \\
von Immunität gegen & ningprogramm teilgenom- \\
SARS-CoV-2 in einer & men haben, das von haus- \\
Kinderkohorte (Fr1da & ärztlichen KinderärztInnen \\
plus: Typ-1-Diabetesri- & während der Vorsorgeun- \\
siko: Früh erkennen - & tersuchungen angeboten \\
Früh gut behandeln) & wurde
\end{tabular}

Zeitraum

Untersuchte

\section{Dualer (RBD}

Anti-S, Anti-N)

LIPS (nicht kom-

merziell)

$01 / 20-08 / 20$

$09 / 20-02 / 21$

$01 / 21-02 / 21$

Stichprobe

15.523 Kinder

11.380 Kinder

11.380 Kinder

Alter in

Jahren

KI)

1-10

$0,7 \%(0,6-0,8)$

$1-10$

$1-5$

$3,9 \%(3,6-4,3)$

6-10

$5,6 \%(4,7-6,7)$

$8,4 \%(6,4-10,9)$

tersuchungen angeboten

wurde

in Bayern (2019-2022;

[14])

Freder1k-Studie:

Typ-1-Diabetesrisiko

früh erkennen ([15];

Bayern)

Einladung aller Kinder, die am Diabetes-Typ-1-Screeningprogramm teilgenommen haben, das von hausärztlichen KinderärztInnen während der Vorsorgeuntersuchungen angeboten wurde

CoKiBa: Coronavirus bei Kindern in Bayern ([19]; 3 Regionen mit hohem, mittlerem und niedrigem Infektionsgeschehen)
Einladung aller Kinder mit einem Termin zu einer Vorsorgeuntersuchung 2020 in teilnehmenden Kinderarztpraxen

\begin{tabular}{llll|l} 
Dualer (RBD & $04 / 20-06 / 20$ & 1916 Kinder & $\begin{array}{l}\text { Neugebo- } \\
\text { rene }\end{array}$ & $0,5 \%$ \\
Anti-S, Anti-N) & & &
\end{tabular}

LIPS (nicht kommerziell)

Elecsys Anti-N
Pan-Ig Roche,
inhouse ELISA
(RBD Anti-S-IgG)

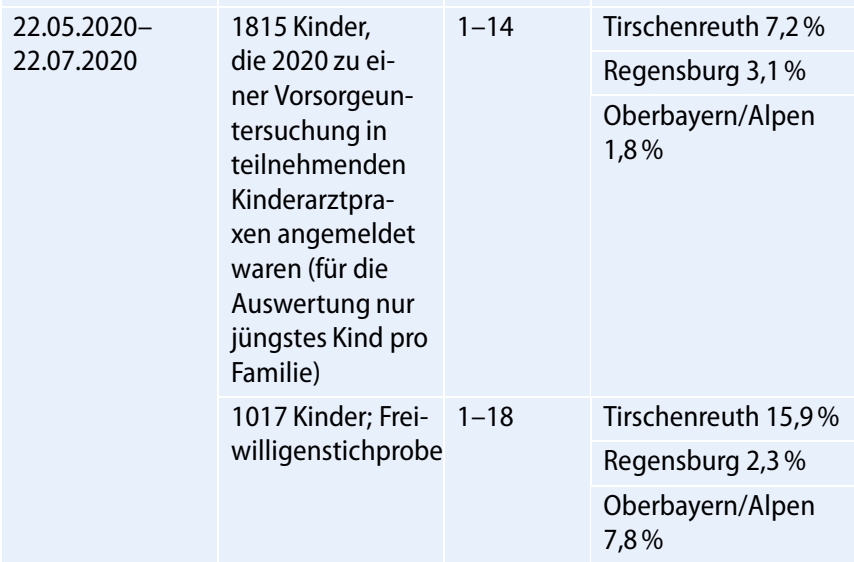
$7,8 \%$

ELISA Enzyme-linked Immunosorbent Assay, IgG Immunglobulin G, KI Konfidenzintervall, LIPS Luciferase-Immunopräzipitations-Test, RBD Rezeptorbindungsdomäne

viele SARS-CoV-2-infizierte Kinder gab, wie für diesen Zeitraum nach Infektionsschutzgesetz an die Gesundheitsämter gemeldet wurden. Die Untererfassung sank, den Ergebnissen der Frlda-Studie nach, in der zweiten Jahreshälfte 2020 bis Anfang 2021 auf den Faktor 3 bis 4 (bei Erwachsenen sank dieser Faktor noch deutlicher auf etwa den Faktor 2; [11, 14]).

\section{Diskussion}

\section{Seroepidemiologische Studien}

Bis Anfang September 2021 (KW 35) sind dem Robert Koch-Institut insgesamt 681.288 laborbestätigte SARS-CoV2-Fälle für 0- bis 19-Jährige übermittelt worden. ${ }^{3}$ Es werden jedoch nicht alle Infektionen labordiagnostisch abgeklärt, wenn sie beispielsweise unbemerkt oder mit milden und unspezifischen Symptomen ablaufen. Da Kinder und Jugendliche sogar häufiger als Erwachsene asymptomatische oder mild symptomatische SARS-CoV-2-Infektionen haben, stellen seroepidemiologische Studien trotz der angesprochenen Limitationen eine essenzielle Datenbasis für die Beurteilung des Infektionsgeschehens dar.

Ergebnisse seroepidemiologischer SARS-CoV-2-Studien in Deutschland, die sich auf Kinder und Jugendliche konzentrieren, liegen bislang erst in geringem Umfang basierend auf lokal-regionalen, nicht repräsentativen Stichproben und

\footnotetext{
3 http://www.rki.de/covid-19-altersverteilung.
}

nur bis maximal Februar 2021 vor. Hier kommt zum Tragen, dass bei Kindern und Jugendlichen vor der Durchführung von Studien, die mit einer Blutentnahme verbunden sind, eine Reihe von Herausforderungen bewältigt werden müssen, die vom Stichprobenverfahren, der $\mathrm{Zu}$ stimmung von Ethikkommissionen, der Zulassung von Beprobungsmaterial und Tests bis hin zur Teilnahmebereitschaft von Eltern und Kindern reichen. Die Studien, für die bislang Ergebnisse vorliegen, erlauben in der Gesamtschau noch keine repräsentativen und nach Setting und Altersgruppen belastbaren Aussagen und können zunächst nur grob die Größenordnung des Anteils von bislang mit SARS-CoV-2 infizierten Kindern und Jugendlichen anzeigen. Diese Größenordnung steht im Einklang mit 
Tab. 2 Seroepidemiologische Studien bzgl. SARS-CoV-2 bei Kindern und Jugendlichen mit Freiwilligenstichproben (Nichtzufallsstichproben/ Convenience Samples) in Wohnbevölkerung, Kinderklinik, Schule und Kita bzw. Vorschule

\begin{tabular}{|c|c|c|c|c|c|c|}
\hline Studie & Stichprobenrahmen & Antikörpertests & Zeitraum & $\begin{array}{l}\text { Untersuchte } \\
\text { Stichprobe }\end{array}$ & $\begin{array}{l}\text { Alter in Jah- } \\
\text { ren }\end{array}$ & $\begin{array}{l}\text { Seroprävalenz } \\
(95 \%-K I)\end{array}$ \\
\hline \multicolumn{7}{|l|}{ Wohnbevölkerung } \\
\hline \multirow{3}{*}{$\begin{array}{l}\text { Prävalenz von } \\
\text { COVID-19 bei Kin- } \\
\text { dern in Baden-Würt- } \\
\text { temberg TEIL A [22] }\end{array}$} & \multirow{3}{*}{$\begin{array}{l}\text { Elternteil-Kind-Paare ohne labor- } \\
\text { bestätigte COVID-19-Diagnose, } \\
\text { Rekrutierung über Zeitungen und } \\
\text { soziale Medien; Einladung in die } \\
\text { Unikliniken Freiburg, Heidelberg, } \\
\text { Tübingen und Ulm }\end{array}$} & \multirow{3}{*}{$\begin{array}{l}\text { Euroimmun } \\
\text { S1-IgG, Immu- } \\
\text { nofluoreszens- } \\
\text { test + ECLIA, ELISA } \\
\text { oder inhouse lu- } \\
\text { minex-basierter } \\
\text { Assay }\end{array}$} & \multirow{3}{*}{$\begin{array}{l}22.04 .2020- \\
15.05 .2020\end{array}$} & \multirow{3}{*}{$\begin{array}{l}2482 \text { Kinder } \\
\text { (+2482 Elternteile) }\end{array}$} & $1-10$ & $0,6 \%(0,3-1,0)$ \\
\hline & & & & & $1-5$ & $0,5 \%(0,2-0,9)$ \\
\hline & & & & & $6-10$ & $0,7 \%(0,4-1,4)$ \\
\hline \multicolumn{7}{|l|}{ Kinderklinik } \\
\hline \multirow{3}{*}{$\begin{array}{l}\text { C19.CHILD Hamburg } \\
\text { [23] }\end{array}$} & \multirow{3}{*}{$\begin{array}{l}\text { Stationäre und ambulante Patient- } \\
\text { Innen aller Hamburger Kinder- } \\
\text { kliniken, der Wohnbevölkerung } \\
\text { sowie Teilnehmende anderer lau- } \\
\text { fender Studien }\end{array}$} & \multirow{3}{*}{$\begin{array}{l}\text { ECLIA Roche und } \\
\text { DiaSorin }\end{array}$} & \multirow{3}{*}{$\begin{array}{l}\text { 11.05.2020- } \\
05.06 .2020\end{array}$} & \multirow{3}{*}{$\begin{array}{l}2436 \text { Kinder und } \\
\text { Jugendliche }\end{array}$} & $0-18$ & $1,2-1,5 \%$ \\
\hline & & & & & $0-9$ & $1 \%$ \\
\hline & & & & & $10-18$ & $2 \%$ \\
\hline \multicolumn{7}{|l|}{ Schule } \\
\hline \multirow{5}{*}{$\begin{array}{l}\text { SCHULERHEBUNG } \\
\text { "CORONA“: Studie } \\
\text { zur Bewertung des } \\
\text { Infektionsgeschehens } \\
\text { mit SARS-CoV-2 bei } \\
\text { Lehrkräften, Schüle- } \\
\text { rinnen und Schülern } \\
\text { in Sachsen ([17]; Leip- } \\
\text { zig, Dresden, Borna, } \\
\text { Werdau, Zwickau) }\end{array}$} & \multirow{5}{*}{$\begin{array}{l}\text { Schülerlnnen und Lehrpersonal } \\
\text { aus } 18 \text { Schulen in } 5 \text { sächsischen } \\
\text { Städten }\end{array}$} & \multirow[t]{5}{*}{-} & $05 / 20-06 / 20$ & $\begin{array}{l}2344 \text { Kinder } \\
\text { und Jugendli- } \\
\text { che + Erwachsene }\end{array}$ & $7-67$ & $0,6 \%$ \\
\hline & & & $09 / 20$ & $\begin{array}{l}2191 \text { Kinder } \\
\text { und Jugendli- } \\
\text { che + Erwachsene }\end{array}$ & $7-67$ & $0,6 \%$ \\
\hline & & & \multirow[t]{3}{*}{$11 / 20$} & \multirow{3}{*}{$\begin{array}{l}2194 \text { Kinder } \\
\text { und Jugendli- } \\
\text { che + Erwachsene }\end{array}$} & $7-67$ & $1,4 \%$ \\
\hline & & & & & $\begin{array}{l}\text { Grundschul- } \\
\text { kinder }^{\mathrm{a}}\end{array}$ & $0,5 \%$ \\
\hline & & & & & Jugendliche ${ }^{a}$ & $1,8 \%$ \\
\hline \multirow{4}{*}{$\begin{array}{l}\text { SchoolCoviDD19 } \\
\text { ([18]; Ostsachsen) }\end{array}$} & \multirow{4}{*}{$\begin{array}{l}\text { Schülerlnnen der Klassen 8-11 so- } \\
\text { wie Lehrpersonal aus } 13 \text { Schulen }\end{array}$} & \multirow{4}{*}{$\begin{array}{l}\text { DiaSorin Liai- } \\
\text { son S1/S2 IgG } \\
\text { CLIA+CMIA Ab- } \\
\text { bott (Anti-N), } \\
\text { Euroimmun S1- } \\
\text { IgG }\end{array}$} & \multirow{2}{*}{$\begin{array}{l}25.05 .2020 \\
30.06 .2020\end{array}$} & 1538 Jugendliche & $14-16$ & $0,7 \%$ \\
\hline & & & & 507 Lehrkräfte & $37-57$ & $0,2 \%$ \\
\hline & & & \multirow{2}{*}{$\begin{array}{l}15.09 .2020- \\
13.10 .2020\end{array}$} & 1334 Jugendliche & $14-16$ & $0,8 \%$ \\
\hline & & & & 445 Lehrkräfte & $36-57$ & $0,2 \%$ \\
\hline \multirow[t]{4}{*}{$\begin{array}{l}\text { BECOSS Schule: Berli- } \\
\text { ner Corona Schulstu- } \\
\text { die }[16,20]\end{array}$} & \multirow{4}{*}{$\begin{array}{l}\text { Jeweils } 20 \text { Schülerlnnen und } \\
10 \text { LehrerInnen aus } 24 \text { Berliner } \\
\text { Schulen; Klassen 3-5, 9-11; Schul- } \\
\text { auswahl über geschichtetes Zu- } \\
\text { fallsverfahren nach Gesundheits- } \\
\text { und Sozialstrukturatlas }\end{array}$} & \multirow{4}{*}{$\begin{array}{l}\text { Euroimmun S1- } \\
\text { IgG }\end{array}$} & $\begin{array}{l}11.06 .2020- \\
19.06 .2020\end{array}$ & $\begin{array}{l}385 \text { Kinder und } \\
\text { Jugendliche (+150 } \\
\text { Personal) }\end{array}$ & $8-18$ & $1,3 \%$ \\
\hline & & & \multirow[t]{3}{*}{$\begin{array}{l}02.11 .2020- \\
16.11 .2020\end{array}$} & $\begin{array}{l}352 \text { Kinder und } \\
\text { Jugendliche }\end{array}$ & $9-18$ & $2,0 \%(0,8-4,1)$ \\
\hline & & & & 142 Personal & $28-65$ & $1,4 \%(0,2-5,0)$ \\
\hline & & & & $\begin{array}{l}625 \text { Familienange- } \\
\text { hörige }\end{array}$ & $2-86$ & $1,4 \%(0,6-2,7)$ \\
\hline \multicolumn{7}{|l|}{ Kita/Vorschule } \\
\hline BECOSS Kita: Berliner & Jeweils 20 Kinder und 5 Kitamit- & Euroimmun S1- & 28.09.2020- & 155 Kinder & $1-6$ & $0 \%$ \\
\hline $\begin{array}{l}\text { Corona Kitastudie } \\
\text { [21] }\end{array}$ & $\begin{array}{l}\text { arbeitende aus } 12 \text { Berliner Kitas; } \\
\text { Kitaauswahl über geschichtetes } \\
\text { Zufallsverfahren nach Gesund- } \\
\text { heits- und Sozialstrukturatlas }\end{array}$ & $\lg G$ & 02.10 .2020 & $\begin{array}{l}78 \text { Personal, } 487 \mathrm{Fa}- \\
\text { milienangehörige }\end{array}$ & $18-78$ & $0,2 \%$ \\
\hline
\end{tabular}


den Ergebnissen für vergleichbare Zeitpunkte bei Erwachsenen in Deutschland, d.h. mit Seroprävalenzen im niedrigen einstelligen Bereich außerhalb ausgewiesener Hotspots.

\section{Untererfassung von infizierten Kindern und Jugendlichen}

Zur Abschätzung der Untererfassung (Dunkelziffer) ist ein hoher Grad an Repräsentativität notwendig, der bei vielen Stichprobenzugängen der hier dargestellten Studien nicht beurteilbar ist. In der Fr1da-Studie wurde die Untererfassung unter der Annahme berechnet, dass die am Diabetesscreening teilnehmenden, überwiegend jüngeren Kinder bezüglich des Infektionsrisikos der Gesamtheit der Kinder in Deutschland ähnlich sind. Demnach hätte es bei jüngeren Kindern in der ersten Pandemiephase 6-mal so viele mit SARS-CoV-2 infizierte Kinder gegeben, wie für diesen Zeitraum nach Infektionsschutzgesetz an die Gesundheitsämter gemeldet wurden, d.h., die Untererfassung wäre ähnlich wie bei Erwachsenen. Allerdings legt der höhere Anteil an asymptomatischen Infektionen bei Kindern im Zusammenhang mit der überwiegend symptombezogenen Testung im Jahr 2020 eine höhere Untererfassung bei Kindern nahe, was auch durch Ergebnisse der TiKoCo-Studie bestätigt wird, die bei Untersuchung einer geschichteten Zufallsstichprobe der Allgemeinbevölkerung ab 14 Jahren des Landkreises Tirschenreuth bis Juli 2020 in der jüngsten Altersgruppe der 14- bis 19-Jährigen die höchste Untererfassung (Faktor 12) berechnet hat [24].

\section{Vergleich mit weiteren Studien bei Erwachsenen, die Kinder miteinschließen}

In der vorliegenden Übersicht nicht enthalten sind die bislang wenigen seroepidemiologischen Studien mit dem Fokus Erwachsene, die auch Kinder und Jugendliche eingeschlossen haben. Die Ergebnisse der KoCo19-Studie in Münchener Haushalten mit Teilnehmenden ab 14 Jahren zeigen für die Altersgruppe der 14- bis 19-Jährigen im Frühjahr 2020 eine SARS-CoV-2-Seroprävalenz von ca. 1,5\%. In der zweiten Runde der Münchener KoCo19-Studie von November 2020 bis Januar 2021 betrug die Seroprävalenz bei 0- bis 19-Jährigen 4,3\% ( $n=212$; [25]). Höhere Prävalenzen sind aus den sogenannten Hotspotstudien plausibel, unter denen beispielsweise die TiKoCo-Studie in Tirschenreuth Teilnehmende ab 14 Jahren eingeschlossen hat. Für das Frühjahr 2020 weist TiKoCo eine Seroprävalenz für die Altersgruppe $14-19$ Jahre von $10 \%$ aus $(n=227$ Untersuchte in der Altersgruppe; [24]). Ebenfalls in Tirschenreuth hatte im Sommer 2020 die CoKiBa-Studie eine Seroprävalenz von $7 \%$ bei Jugendlichen und von $5 \%$ bzw. $6 \%$ für Kinder im Kitaund Grundschulalter ermittelt. Wenig überraschend ist, dass die Seroprävalenz noch einmal höher war in der Unterstichprobe der Teilnehmenden, die nicht aufgrund einer Vorsorgeuntersuchung, sondern anlassbezogen die Kinderarztpraxis aufgesucht hatten und getestet wurden [19].

\section{Vergleich mit internationalen Studien}

Auch international sind weit weniger seroepidemiologische SARS-CoV-2Studien mit dem Fokus Kinder als mit dem Fokus Erwachsene durchgeführt worden [26]. Eine der ersten großen nationalen bevölkerungsbezogenen seroepidemiologischen Studien, die ENECOVID-Studie aus Spanien, berichtete eine Seroprävalenz von unter $3 \%$ bei Kindern von 1-9 Jahren während der ersten Pandemiewelle [27]. Altersstratifizierte, für die Allgemeinbevölkerung aussagekräftige Seroprävalenzen von SARSCoV-2 wurden auch in den Niederlanden während der ersten Pandemiewelle ermittelt. Danach betrug die Seroprävalenz bei Kindern von 2-17 Jahren 1,7\% [28]. Schätzungen für die Prävalenz von SARS-CoV-2-Antikörpern für das Frühjahr 2020 wurden auch für die französische Bevölkerung präsentiert. Die Untersuchung von Restseren im Zeitraum März-Mai 2020 ergab eine landesweite Seroprävalenz bei Kindern unter 10 Jahren von $3 \%$ [29]. Eine Studie aus Dänemark aus dem Sommer 2020 ergab eine Seropositivität für Kinder und Jugendliche von 0-17 Jahren von 1,6\% [30]. Die SEROCoV-POP-Studie vom Frühjahr 2020 im Kanton Genf, Schweiz, berichtete für die Altersgruppe der 5- bis 9-Jährigen eine Seroprävalenz von $0,8 \%$ [31]. In Wien, Österreich, ergab eine von Mai bis Juli 2020 durchgeführte Studie bei Schülerinnen und Schülern (5-21 Jahre) eine SARS-CoV2-Seroprävalenz von insgesamt $1,3 \%$ [32]. Bei Kindergartenkindern im Alter von 5 Monaten bis 4 Jahren in Frankreich (einschließlich Paris, das zu der Zeit Hochinzidenzgebiet war) wurde eine Seroprävalenz von $4 \%$ gezeigt [33].

Ähnlich wie die CoKiBa-Studie aus Baden-Württemberg im Setting Kinderarztpraxis berichtete auch eine landesweite Studie aus Portugal, die zwischen Mai und Juli 2020 Teilnehmende über ein Netzwerk von Laboratorien für klinische Pathologie und durch öffentliche Krankenhäuser rekrutierte, höhere SARSCoV-2-Seroprävalenzen von $8 \%$ und $10 \%$ für die Altersgruppen 1-9 Jahre beziehungsweise 10-19 Jahre [34]. Eine ähnliche Gesamtseroprävalenz von 9,5\% berichtete eine von Juli-Oktober 2020 durchgeführte Studie aus dem Großraum Washington, USA, bei pädiatrischen $\mathrm{Pa}$ tientinnen und Patienten im Alter von 2 Monaten bis 22 Jahren [35]. In einer Studie bei 0 - bis 15 -Jährigen in 27 Kinderarztpraxen im Großraum Paris, einem Gebiet, das stark von COVID-19 betroffen war, betrug nach dem Höhepunkt der ersten Pandemiewelle die Rate der Seropositivität 10,7\% [36]. In der RAPID19-Studie bei 2- bis 15-jährigen Kindern von Gesundheitspersonal in 5 Regionen im Vereinigten Königreich (London, Belfast, Cardiff, Manchester, Glasgow) lag die Seroprävalenz für April-Juli 2020 bei $7 \%$ [37].

Für die 2. Pandemiewelle lassen sich national wie international höhere Prävalenzen von SARS-CoV-2-Antikörpern feststellen. Eine Studie bei Kindern und Jugendlichen $<18$ Jahren im Bundesstaat Mississippi, USA, ermittelte eine Seropositivität von $2,5 \%$ im Mai 2020 und $16,3 \%$ im September 2020 [38]. Eine Studie aus Belgien im Herbst 2020 stellte für 6- bis 15-Jährige eine Seroprävalenz von $4 \%$ in einer Region mit niedriger Transmission und $14 \%$ in einer Region mit 
hoher Transmission fest [39]. Die 2. Untersuchungswelle der SEROCoV-POPStudie aus Genf (November-Dezember 2020) ermittelte eine Seroprävalenz bei Kindern im Alter von 0-5 Jahren von $15 \%$ [40]. Auch die Schweizer Studie „Ciao Corona“ bei Kindern im Alter von 6-16 Jahren der Klassen 1-2, 4-5 und 7-8 aus 55 zufällig ausgewählten Schulen in der Region Zürich wurde als longitudinale Untersuchung durchgeführt. Vor dem Hintergrund, dass die Schulen in der Schweiz seit Beginn des neuen Schuljahres im August 2020 bis Jahresende nicht geschlossen wurden, lag die Gesamt-SARS-CoV-2-Seroprävalenz im Sommer bei $2,4 \%$ und im Spätherbst bei $4,5 \%$ der zuvor nicht seropositiven Kinder, was zu geschätzten 7,8\% jemals seropositiven Kindern führte [41].

\section{Ausblick}

Seroepidemiologische Studien zu SARSCoV-2 bei Kindern werden auch im weiteren Verlauf der Pandemie von Interesse sein. Es gilt dabei, zum einen abzuschätzen, welcher Anteil der Kinder und Jugendlichen bereits eine Infektion hatte oder geimpft ist. Zum anderen gilt es, die Verbreitung körperlicher und psychischer Beeinträchtigungen im Nachgang einer Infektion zu untersuchen. Um Ergebnisse solcher Studien auf unselektierte Kinder und Jugendliche in Deutschland übertragen zu können, ist eine weitere Optimierung der Stichprobenzugänge anzustreben. Dies gilt insbesondere für den Stichprobenzugang Vorsorgeuntersuchung, da beispielsweise Kinder und Jugendliche aus Familien mit niedrigem Sozialstatus seltener an Früherkennungsuntersuchungen teilnehmen als Gleichaltrige aus Familien mit hohem Sozialstatus [42].

Die Unterscheidung von Antikörpern nach Infektion und nach Impfung ist prinzipiell möglich, hat aber Limitationen. Gegen das Spike-Protein (AntiS) gerichtete Antikörper sind sowohl nach einer Infektion als auch nach einer Impfung nachzuweisen, während Antikörper gegen das Nukleokapsid-Protein (Anti-N) sich nur nach einer Infektion mit SARS-CoV-2 entwickeln. Allerdings sind Antikörper keine über die Zeit konstanten Marker einer stattgehabten Infektion. Außerdem unterscheiden sich Verläufe von Antikörperspiegeln im Serum je nach Zielstruktur der Antikörper (Anti-S, Rezeptorbindungsdomäne [RBD] oder Anti-N; [43, 44]). Sowohl initial als auch im weiteren zeitlichen Verlauf nach einer Infektion weichen Antikörpertests verschiedener Hersteller zum Teil sehr deutlich in ihren Testgütekriterien, d.h. ihrer Sensitivität und Spezifität, voneinander ab [45, 46]. Zudem steigen die Antikörperspiegel nach einer asymptomatischen Infektion oder einem milden Krankheitsverlauf schon initial weniger hoch an als bei mittleren oder schweren Krankheitsverläufen [47, 48]. Hinzu kommt das Phänomen des sogenannten Waning bis hin zur Seroreversion, also dem im Zeitverlauf nach einer Infektion (oder Impfung) fortschreitenden Abfall von Antikörperspiegeln unter den Testgrenzwert [49]. Eine Adjustierung für Waning und Seroreversion wird mit länger werdenden Zeiträumen zwischen Studienfeldphase und Zeitpunkt der Infektion immer bedeutsamer werden [50].

Ergebnisse weiterer seroepidemiologischer Studien mit Fokus Kinder und Jugendliche in Deutschland werden in Kürze erwartet. Geplante Studien, die durch Pressemitteilungen, Registrierung von Studienprotokollen oder über Netzwerke und persönliche Mitteilungen bekannt werden, werden zeitnah in die fortlaufende Übersicht auf der Webseite des Robert Koch-Instituts aufgenommen. Dies ermöglicht die weitere Vernetzung, den Austausch und die zeitnahe Aktualisierung der hier vorliegenden Ergebnisübersicht.

\section{Korrespondenzadresse}

\section{Dr. Roma Thamm}

Abteilung Epidemiologie und Gesundheitsmonitoring, Robert Koch-Institut

General-Pape-Str. 62-66, 12101 Berlin,

Deutschland

ThammR@rki.de

Funding. Open Access funding enabled and organized by Projekt DEAL.

\section{Einhaltung ethischer Richtlinien}

Interessenkonflikt. R. Thamm, N. Buttmann-Schweiger, J. Fiebig, C. Poethko-Müller, F. Prütz, G. Sarganas und $\mathrm{H}$. Neuhauser geben an, dass kein Interessenkonflikt besteht.

Für diesen Beitrag wurden von den Autoren keine Studien an Menschen oder Tieren durchgeführt. Für die aufgeführten Studien gelten die jeweils dort angegebenen ethischen Richtlinien.

Open Access. Dieser Artikel wird unter der Creative Commons Namensnennung 4.0 International Lizenz veröffentlicht, welche die Nutzung, Vervielfältigung, Bearbeitung, Verbreitung und Wiedergabe in jeglichem Medium und Format erlaubt, sofern Sie den/die ursprünglichen Autor(en) und die Quelle ordnungsgemäß nennen, einen Link zur Creative Commons Lizenz beifügen und angeben, ob Änderungen vorgenommen wurden.

Die in diesem Artikel enthaltenen Bilder und sonstiges Drittmaterial unterliegen ebenfalls der genannten Creative Commons Lizenz, sofern sich aus der Abbildungslegende nichts anderes ergibt. Sofern das betreffende Material nicht unter der genannten Creative Commons Lizenz steht und die betreffende Handlung nicht nach gesetzlichen Vorschriften erlaubt ist, ist für die oben aufgeführten Weiterverwendungen des Materials die Einwilligung des jeweiligen Rechteinhabers einzuholen.

Weitere Details zur Lizenz entnehmen Sie bitte der Lizenzinformation auf http://creativecommons.org/ licenses/by/4.0/deed.de.

\section{Literatur}

1. Amanat F, Stadlbauer D, Strohmeier $S$ et al (2020) A serological assay to detect SARSCoV-2 seroconversion in humans. Nat Med 26:1033-1036. https://doi.org/10.1038/s41591020-0913-5

2. Corman VM, Landt $O$, Kaiser $M$ et al (2020) Detection of 2019 novel coronavirus (2019-nCoV) by real-time RT-PCR. Euro Surveill. https://doi.org/ 10.2807/1560-7917.es.2020.25.3.2000045

3. Euroimmun Pressemitteilung (2020) Tests für den Nachweis von Antikörpern gegen SARS-CoV-2 ab sofort verfügbar. https://www.euroimmunblog. de/tests-fuer-den-nachweis-von-antikoerperngegen-sars-cov-2-ab-sofort-verfuegbar/. Zugegriffen: 20. Mai 2021

4. CDC COVID-19 Response Team (2020) Coronavirus disease 2019 in children-United States, february 12-april 2, 2020. Mmwr Morb Mortal Wkly Rep 69:422-426. https://doi.org/10.15585/mmwr. $\mathrm{mm} 6914 \mathrm{e} 4$

5. Dong Y, Mo X, Hu Y et al (2020) Epidemiology of COVID-19 among children in China. Pediatrics 145(6):e20200702. https://doi.org/10.1542/peds. 2020-0702

6. European Centre for Disease Prevention and Control (2020) COVID-19 in children and the role of school settings in transmission-first update. https://www.ecdc.europa.eu/en/publicationsdata/children-and-school-settings-covid-19transmission. Zugegriffen: 20. Mai 2021

7. lannarella R, Lattanzi C, Cannata G et al (2020) Coronavirus infections in children: from SARS 
and MERS to COVID-19, a narrative review of epidemiological and clinical features. Acta Biomed 91:e2020032. https://doi.org/10.23750/ abm.v91i3.10294

8. Riccardo F, Ajelli M, Andrianou XD et al (2020) Epidemiological characteristics of COVID-19 cases and estimates of the reproductive numbers 1 month into the epidemic, Italy, 28 January to 31 March 2020. Euro Surveill 25:2000790. https://doi. org/10.2807/1560-7917.es.2020.25.49.2000790

9. Hoste L, Van Paemel R, Haerynck F (2021) Multisystem inflammatory syndrome in children related to COVID-19: a systematic review. Eur J Pediatr 180:2019-2034. https://doi.org/10.1007/ s00431-021-03993-5

10. Zeichner SL, Cruz AT (2020) Multisystem inflammatory syndrome in children and SARS-coV-2 serology. Pediatrics 146:e2020032888. https://doi. org/10.1542/peds.2020-032888

11. Neuhauser $H$, Buttmann-Schweiger $N$, Ellert $U$ et al (2021) Seroepidemiologische Studien zu SARSCoV-2 in Stichproben der Allgemeinbevölkerung und bei Blutspenderinnen und Blutspendern in Deutschland - Ergebnisse bis August 2021. Epidemiol Bull 37:3-12. https://doi.org/10.25646/ 8999

12. Poethko-Müller C, Prütz F, Buttmann-Schweiger N et al (2020) Studien zur Seroprävalenz von SARSCoV-2 in Deutschland und international. J Health Monit 5:2-16. https://doi.org/10.25646/7023

13. Schilling J, Buda S, Fischer M et al (2021) Retrospektive Phaseneinteilung der COVID-19Pandemie in Deutschland bis Februar 2021. Epidemiol Bull 15:8-17. https://doi.org/10.25646/ 8149

14. Hippich M, Sifft P, Zapardiel-Gonzalo J et al (2021) A public health antibody screening indicates a marked increase of SARS-CoV-2 exposure rate in children during the second wave. Med (N Y) 2:571-572. https://doi.org/10.1016/j.medj.2021. 03.019

15. Hippich M, Holthaus L, Assfalg R et al (2021) A public health antibody screening indicates a 6 -fold higher SARS-coV-2 exposure rate than reported cases in children. Med (NY) 2:149-163. https://doi. org/10.1016/j.medj.2020.10.003

16. Hommes $F$, van Loon $W$, Thielecke $M$ et al (2021) SARS-CoV-2 infection, risk perception, behaviour and preventive measures at schools in Berlin, Germany, during the early post-lockdown phase: a cross-sectional study. Int J Environ Res Public Health 18:2739. https://doi.org/10.3390/ ijerph18052739

17. Jurkutat A, Meigen C, Vogel M, Kiess W (2020) Studie zur Bewertung des Infektionsgeschehens mit SARS-CoV-2 bei Lehrkräften, Schülerinnen und Schülern in Sachsen. https://home.unileipzig.de/lifechild/wp-content/uploads/2020/ 12/Kurzbericht_nach-Novembertests_final.pdf. Zugegriffen:21. Sept. 2021

18. Kirsten C, Unrath M, Luck C, Dalpke AH, Berner R, Armann J (2021) SARS-coV-2 seroprevalence in students and teachers: a longitudinal study from may to october 2020 in German secondary schools. BMJ Open 11:e49876. https://doi.org/10.1136/ bmjopen-2021-049876

19. LaubO,LeipoldG, Toncheva Aetal(2020) Coronavirus antibody screening identies children with mild to moderate courses of PMIS. Authorea. https:// doi.org/10.22541/au.160776187.78854537/v1

20. Theuring S, Thielecke $M$, van Loon W et al (2021) SARS-CoV-2 infection and transmission in school settings during the second COVID-19 wave: a crosssectional study, Berlin, Germany, November 2020.
Euro Surveill 26:2100184. https://doi.org/10.2807/ 1560-7917.ES.2021.26.34.2100184

21. Thielecke $M$, Theuring $S$, van Loon $W$ et al (2021) SARS-CoV-2 infections in kindergartens and associated households at the start of the second wave in Berlin, Germany - a cross sectional study. Eur J Public Health. https://doi.org/10.1093/ eurpub/ckab079

22. Tönshoff B, Müller B, Elling R et al (2021) Prevalence of SARS-CoV-2 infection in children and their parents in southwest Germany. JAMA Pediatr 175:586-593. https://doi.org/10.1001/ jamapediatrics.2021.0001

23. UKE Hamburg Pressemitteilung (2020) C19.CHILD Hamburg: Ältere Kinder haben häufiger Antikörper gegen SARS-CoV-2. https://www. uke.de/allgemein/presse/pressemitteilungen/ detailseite_95874.html.Zugegriffen:20.Mai 202

24. Wagner R, Peterhoff D, Beileke $S$ et al (2021) Estimates and determinants of SARS-CoV-2 seroprevalence and infection fatality ratio using latent class analysis: the population-based Tirschenreuth study in the hardest-hit German county in spring $2020 \mathrm{https} / / /$ doi.org/10.1101/ 2021.03.29.21254343 (medRxiv)

25. Radon K, Bakuli A, Pütz P et al (2021) From first to second wave: follow-up of the prospective COVID 19 cohort (KoCo19) in Munich (Germany). BMC InfectDis 21:925. https://doi.org/10.1186/s12879021-06589-4

26. Bobrovitz N, Arora RK, Cao C et al (2021) Global seroprevalence of SARS-CoV-2 antibodies: a systematic review and meta-analysis https://doi. org/10.1101/2020.11.17.20233460 (medRxiv)

27. Pollan M, Perez-Gomez B, Pastor-Barriuso R et al (2020) Prevalence of SARS-CoV-2 in Spain (ENE-COVID): a nationwide, population-based seroepidemiological study. Lancet 396:535-544. https://doi.org/10.1016/S0140-6736(20)31483-5

28. Vos ERA, den Hartog G, Schepp RM et al (2020) Nationwide seroprevalence of SARS-CoV-2 and identification of risk factors in the general population of the Netherlands during the first epidemic wave. J Epidemiol Community Health. https://doi.org/10.1136/jech-2020-215678

29. Le Vu S, Jones G, Anna F et al (2020) Prevalence of SARS-CoV-2 antibodies in France: results from nationwide serological surveillance https:/doi. org/10.1101/2020.10.20.20213116 (MedRxiv)

30. RytterMJH,NygaardU, MandicINetal (2021) Prevalence of SARS-CoV-2-antibodies in Danish children and adults. Pediatr Infect Dis J 40:e157-e159. https://doi.org/10.1097/INF.0000000000003048

31. Stringhini S, Wisniak A, Piumatti G et al (2020) Seroprevalence of anti-SARS-CoV-2 lgG antibodies in Geneva, Switzerland (SEROCoV-POP): a population-based study. Lancet. https://doi.org/10.1016/ s0140-6736(20)31304-0

32. Szepfalusi Z, Schmidthaler K, Sieber J et al (2021) Lessons from low seroprevalence of SARS-CoV-2 antibodies in schoolchildren: a cross-sectional study. Pediatr Allergy Immunol 32:762-770. https://doi.org/10.1111/pai.13459

33. Lachassinne $E$, de Pontual $L$, Caseris $M$ et al (2021) SARS-CoV-2 transmission among children and staff in daycare centres during a nationwide lockdown in France: a cross-sectional, multicentre, seroprevalence study. Lancet Child Adolesc Health 5:256-264. https://doi.org/10.1016/s23524642(21)00024-9

34. Kislaya I, Goncalves P, Barreto $M$ et al (2021) Seroprevalence of SARS-CoV-2 infection in Portugal in may-july 2020: results of the first national serological survey (ISNCOVID-19). Acta
Med Port 34:87-94. https://doi.org/10.20344/ amp.15122

35. Bahar B, Simpson JN, Biddle Cetal (2021) Estimated SARS-CoV-2 seroprevalence in healthy children and those with chronicillnesses in the Washington metropolitan area as of october 2020. Pediatr Infect Dis J 40:e272-e274. https://doi.org/10.1097/INF. 0000000000003140

36. Cohen R, Jung C, Ouldali N et al (2020) Assessment of SARS-CoV-2 infection by reverse transcriptionPCR and serology in the paris area: a cross-sectional study. BMJ Paediatr Open 4:e887. https://doi.org/ 10.1136/bmjpo-2020-000887

37. Waterfield T, Watson C, Moore R et al (2020) Seroprevalence of SARS-CoV-2 antibodies in children: a prospective multicentre cohort study. Arch Dis Child. https://doi.org/10.1136/archdischild-2020320558

38. Hobbs CV, Drobeniuc J, Kittle T et al (2021) Estimated SARS-CoV-2 seroprevalence among persons aged (18 years-Mississippi, May-September 2020. Mmwr Morb Mortal Wkly Rep 70:312-315. https://doi.org/10.15585/mmwr.mm7009a4

39. Boey L, Roelants M, Merckx J et al (2021) Age-dependent seroprevalence of SARS-CoV-2 antibodies in school-aged children from areas with low and high community transmission. Res Sq. https://doi.org/10.21203/rs.3.rs-221830/v1

40. Stringhini S, Zaballa M-E, Perez-Saez J et al (2021) Seroprevalence of anti-SARS-CoV-2 antibodies after the second pandemic peak. Lancet Infect Dis 21:600-601. https://doi.org/10.1016/s14733099(21)00054-2

41. Ulyte A, Radtke T, Abela IA et al (2021) Clustering and longitudinal change in SARS-CoV-2 seroprevalence in school children in the canton of Zurich, Switzerland: prospective cohort study of 55 schools. BMJ 372:n616. https://doi.org/10.1136/ bmj.n616

42. Robert Koch-Institut (Hrsg) (2015) Inanspruchnahme von Früherkennungsuntersuchungen. Faktenblatt zu KiGGS Welle 1: Studie zur Gesundheit von Kindern und Jugendlichen in Deutschland -Erste Folgebefragung 2009-2012. RKI, Berlin

43. Ripperger TJ, Uhrlaub JL, Watanabe M et al (2020) Orthogonal SARS-CoV-2 serological assays enable surveillance of low-prevalence communities and reveal durable humoral immunity. Immunity 53:925-933.e4. https://doi.org/10.1016/j.immuni. 2020.10.004

44. Whitcombe AL, Mcgregor R, Craigie A et al (2020) Comprehensive analysis of SARS-CoV-2 antibody dynamicsin NewZealandhttps://doi.org/10.1101/ 2020.12.10.20246751 (MedRxiv)

45. Harris RJ, Whitaker HJ, Andrews NJ et al (2021) Serological surveillance of SARS-CoV-2: six-month trends and antibody response in a cohort of public health workers. J Infect 82:162-169. https://doi. org/10.1016/j.jinf.2021.03.015

46. Muecksch F, Wise H, Batchelor B et al (2020) Longitudinal analysis of clinical serology assay performance and neutralising antibody levels in COVID19 convalescents https://doi.org/10.1101/ 2020.08.05.20169128 (medRxiv)

47. Long QX, Liu BZ, Deng HJ et al (2020) Antibody responses to SARS-CoV-2 in patients with COVID19. Nat Med 26:845-848. https://doi.org/10.1038/ s41591-020-0897-1

48. Weis S, Scherag A, Baier M et al (2020) Seroprevalence of SARS-CoV-2 antibodies 1 in an entirely PCR-sampled and quarantined community after a COVID-19 outbreak-the CoNAN study https:// doi.org/10.1101/2020.07.15.20154112 (medRxiv) 
49. Ladage $D$, Rösgen $D$, Schreiner $C$ et al (2020) Persisting antibody response to SARS-CoV-2 in a local Austrian population https://doi.org/10. 1101/2020.11.20.20232140 (MedRxiv)

50. Chen S, Flegg JA, White LJ, Aguas R (2021) Levels of SARS-CoV-2 population exposure are considerably higher than suggested by seroprevalence surveys https://doi.org/10.1101/2021.01.08.21249432 (medRxiv) 